\title{
Co-Proceso de combustibles alternos en la industria del cemento: una alternativa para la eliminación de desechos sólidos
}

Co-Process of alternative fuels in the cement industry: an alternative to solid

\author{
Luis Alberto Terán Calderón \\ teran.calderon@gmail.com \\ Código ORCID: 0000-0001-7876-2898
}

Universidad Andina Simón Bolívar,

Sucre - Bolivia

Artículo recibido junio 2020 / Arbitrado en octubre 2020 / Aceptado en octubre 2020 / Publicado en enero 2021

RESUMEN

La investigación tuvo como objetivo analizar a nivel técnico y económico la posibilidad de incorporar un co-proceso de combustibles alternos en la industria del cemento; mediante un estudio mixto de corte transversal que según la etapa tuvo alcance exploratorio, descriptivo o aplicado. Con base en los avances tecnológicos desarrollados en Alemania se estudió las condiciones existentes en el municipio de Sucre - Bolivia, que permitan aprovechar la generación de residuos sólidos en el co-proceso de la cementera local.

Como resultado, se determinó que, en este contexto, la incorporación del coproceso permitiría mantener la calidad actual del cemento; generaría beneficios medio ambientales y de carácter social; tendría un ROI de 13 años y 9 meses. Se concluye que este cambio de combustible en la producción de cemento permitiría la eliminación de residuos sólidos en una sinergia entre el municipio y la industria, que promueva el desarrollo sostenible.

Palabras clave: Proceso; Combustible; Cemento; Desechos sólidos; Basura

ABSTRACT The purpose of the research was to analyze at a technical and economic level the possibility of incorporating a co-process of alternative fuels in the cement industry; through a mixed cross-sectional study that, depending on the stage, had an exploratory, descriptive, or applied scope. Based on the technological advances developed in Germany, the existing conditions in Sucre - Bolivia were studied, which will take advantage of the generation of solid waste in the co-process of the local cement company.

As a result, it was determined that, in this context, the incorporation of the coprocess will allow maintaining the current quality of the cement; it would generate environmental and social benefits; It will have an ROI of 13 years and 9 months. It is concluded that this change of fuel in cement production will allow the elimination of solid waste in a synergy between the municipality and the industry, which promotes sustainable development.

Key words: Process; Fuel; Concrete; Solid waste; Garbage 


\section{INTRODUCCIÓN}

La producción del cemento involucra un alto consumo energético; el proceso propio de transformación del carbonato de calcio $\mathrm{CaCO} 3$ (Caliza) en clinker se realiza a altas temperaturas, en un promedio de $1450{ }^{\circ} \mathrm{C}$; asimismo, se utilizan combustibles fósiles como carbón, petróleo y gas natural (a partir de ahora GN). La disponibilidad de reservas gasíferas en Bolivia hace que el GN sea empleado en la industria cementera nacional. La producción de cemento viene también acompañada de altas emisiones de CO2 (Gante, 2007 \& GoetzNeunhoeffer, 2012), llegando a valores de 0, 825 $\mathrm{t}$ de $\mathrm{CO} 2$ por tonelada de clinker producida (Ludwig, 2013).

El co-proceso en la industria del cemento es una forma de aprovechar la energía calorífica de materiales presentes en los desechos sólidos; asimismo, repercute en el cuidado del medio ambiente fomentando el consumo racional de combustibles fósiles no renovables, sin afectar la calidad del producto final (FLSmidth, 2013).

El municipio de Sucre, con aproximadamente 281000 habitantes al 2017 (INE, 2018), generaba el año 2013 diariamente 170 toneladas de basura (EMAS, 2014). Este alto volumen de materiales sólidos en la actualidad ya ha sobrepasado la capacidad de una eliminación a cielo abierto en botaderos municipales denominados "rellenos sanitarios"; sin un control ni tratamiento adecuado, no exentos de costos económicos, se corre el riesgo de daños al medio ambiente. La administración de estos rellenos sanitarios está a cargo del departamento de Manejo de Residuos Sólido dependiente del gobierno municipal.

Asimismo, la Fábrica Nacional de Cemento (FANCESA) desarrolla actividades industriales en este municipio, con una importante participación en el Producto Interno Bruto (PIB) departamental, y una producción anual de 970 000 tdc (toneladas de cemento) (FANCESA, 2019). Los residuos sólidos (a partir de ahora denominados RS) generados en Sucre contienen materiales con poder calorífico suficiente para ser utilizados como fuente de energía en la producción del cemento.

Esta investigación se enfocó en analizar a nivel técnico y económico la posibilidad de incorporar un co-proceso de combustibles alternos en la industria del cemento en Sucre, como una medida para la eliminación tecnológica de RS. Con este propósito los apartes del artículo científico son: análisis la relación a la generación de RS y la logística de almacenamiento en este municipio; estudio de las variables que intervienen en el co-proceso de combustibles alternos en la industria del cemento; propuesta y análisis técnico y económico del co-proceso. En forma emergente se planteó la necesidad de la valoración de una planta de manejo de RS.

\section{METODOLOGÍA}

Ésta es una investigación de corte transversal y enfoque mixto, con principal aporte cuantitativo que se desarrolló en tres etapas: 1) diagnóstico del municipio de Sucre con relación a la generación de RS; 2) descripción y análisis de las variables que intervienen en el co-proceso en la industria del cemento; 3) análisis técnico y económico de la incorporación de dicho co-proceso.

El diseño metodológico varió según la etapa. La primera etapa requirió del enfoque cualitativo, tuvo alcance descriptivo y aplicó las técnicas de la entrevista, la consulta a expertos y la revisión bibliográfica de la experiencia internacional: Polysius (2008), FLSmidth (2013), Loesche (2009), Bosold y Pickhardt (2014), Duda 
(1985), Braun (2002), Kropp (2012), CEMBUREAU (2009) y Karstensen (2012). La segunda parte tuvo enfoque mixto, alcances exploratorio, descriptivo y analítico, principalmente se aplicaron las técnicas: revisión bibliográfica, para el análisis de la evolución del empleo y grados de sustitución de materias primas alternativas (AFR en inglés) en países que tiene desarrollada esta tecnología; y la observación participativa, para el estudio del proceso productivo de FANCESA. Finalmente, con un enfoque cuantitativo, la tercera etapa tuvo alcance aplicado en cuanto al estudio técnico se refiere y alcance descriptivo en cuanto al análisis económico.

Se hizo un análisis de la evolución del empleo y grados de sustitución de AFR en países que tiene desarrollada esta tecnología. La situación anterior/actual del manejo de RS y su futuro manejo basado en su poder calorífico. Las capacidades de producción y consumo de energía y su relación causa-efecto: volúmenes de combustible y ahorro energético desde la perspectiva económica. En el ámbito local se hizo un análisis de desarrollo tecnológico, consumos energéticos, en la cementera local con base en las memorias anuales publicadas en la última década. Situación similar con el manejo de RS a cargo de Entidad Municipal de Aseo Urbano Sucre (EMAS).

Asimismo, se determinaron los poderes caloríficos de los materiales presentes en los RS de Sucre y la ubicación física óptica para la introducción de AFR en un co-proceso en la industria del cemento. Se desarrolló un análisis matemático de los consumos energéticos en un sistema de calcinación, la evaluación de poderes caloríficos del GN para ser comparados con AFRs y cálculo de costos de la energía consumida.

Por otra parte, el juicio de expertos permitió consultar a profesionales con experiencias teórica y práctica en el área de implementación de co-proceso y ahorro de consumos energéticos. La selección de estos profesionales se basó en la experiencia en el desarrollo e implementación tecnológica de RS en la industria cementera, tales como representantes las empresas alemanas Polysius, Vecoplan y Refratechnik, FLSmidth (USA). El experto de Polysius, gracias a su amplia práctica en evaluación económica, colaboró con los procedimientos de cálculo de rentabilidad económica y el experto de Vecoplan, con el cálculo aproximado de una planta recicladora. Para la administración de los RS se hizo consultas a EMAS.

Las variables de estudio fueron: costo del $\mathrm{GN}$, consumo del GN, temperatura y tiempo de resistencia, granulometría del material, cantidad de RS, calidad de RS, poder calorífico neto de materiales, capacidad de generación de RS, composición de los RS, costo de los RS y RS mayores y tóxicos. Se realizó el análisis matemático de consumos energéticos, costos y volúmenes de sustitución por AFRs.

\section{RESULTADOS Y DISCUSIÓN}

La investigación se sintetiza en tres etapas: la primera se constituye en un diagnóstico del municipio de Sucre con relación a la generación de RS y la logística de almacenamiento; la segunda estuvo destinada a describir las variables que intervienen en el co-proceso de combustibles alternos en la industria del cemento en el contexto de este municipio; la tercera etapa, presenta una propuesta para dicho co-proceso, incluye análisis técnico y económico.

\section{Problemática de los RS en Sucre}

La situación problémica en torno a la generación de residuos en Sucre se 
puntualizada así: El botadero municipal "La Esperanza" ha sobrepasado su capacidad de recepción; se han implementado al alrededor de 247 botaderos clandestinos fuera de toda reglamentación y control sanitario; los residuos líquidos pueden alcanzar corrientes de agua subterránea; los riesgos medioambientales ante situaciones no controladas se incrementan año tras año; existe peligro de explosiones por altas concentraciones de metano en las capas enterradas; las reglamentaciones municipales para separación de desechos sólidos son insuficientes; existe poca motivación para aplicar tecnología en la administración de desechos sólidos; hay desconocimiento del potencial energético/económico en materiales presentes en los desechos sólidos; hay escasa conciencia del cuidado del medio ambiente en la población; existe baja responsabilidad del individuo en el manejo de desechos sólidos.

Por otra parte, la producción de cemento implica: alto consumo energético; altos valores de contaminación de $\mathrm{CO}_{2}$; el combustible utilizado en la industria local es el gas natural (GN), un recurso no renovable; la disponibilidad de este combustible frena el crecimiento tecnológico en la industria del cemento en la región.

\section{Análisis de variables}

Las variables consideradas son las siguientes: costo del GN, consumo del GN, temperatura y tiempo de resistencia, granulometría del material, cantidad de RS, calidad de RS, poder calorífico neto de materiales, capacidad de generación de RS, composición de los RS, costo de los RS y RS mayores y tóxicos. El conjunto de estas variables juega un rol muy importante, permiten alcanzar la utilización máxima del poder calorífico. Se presenta entonces una evaluación técnicoadministrativa de cada variable de interés.

Costo del GN. Variable externa al proceso, principal combustible en las cementeras nacionales dado que Bolivia es productor y exportador de este. Asimismo, tiene un bajo costo comparado con otros países de la región. Es importante también mencionar que el costo interno para el sector industrial tiene una subvención de acuerdo con La Ley de Hidrocarburos que en su Artículo 87 indica que los precios del mercado interno para el GN no podrán sobrepasar el $50 \%$ del precio mínimo del contrato de exportación (HNC, 2005).

Consumo del GN. Para analizar esta variable se consideró una planta con cuatro fuentes de suministro de energía (GN, Carbón, Residuos de Aceites, Neumáticos usados), con dos puntos establecidos para la utilización del combustible primario: quemador principal y quemador en el calcinador (FLSmidth, 2011). La relación de consumo promedio para una línea de producción de 2000 tdc es de $40 \%$ y $60 \%$ respectivamente; asimismo, la relación de consumo promedio para una línea de producción de 760 tdc es de $50 \%$ y $50 \%$ respectivamente (Karstensen, 2012). En líneas generales un consumo referencial de una línea de producción de 2000 tdc está por el orden de $7400[\mathrm{Nm} 3 / \mathrm{h}]$, equivalentes a $4600[\mathrm{Nm} 3 / \mathrm{h}]$ en el quemador principal y $2800[\mathrm{Nm} 3 / \mathrm{h}]$ en el quemador del calcinador. Para una línea de producción de $760[\mathrm{Nm} 3 / \mathrm{h}]$ está por el orden de $2900[\mathrm{Nm} 3 / \mathrm{h}]$, equivalentes a $1400[\mathrm{Nm} 3 / \mathrm{h}]$ en el quemador principal y $1500[\mathrm{Nm} 3 / \mathrm{h}]$ en el quemador del calcinador. Con estos datos se tiene un consumo anual para una producción de 2000 tdc de 53956360 [Nm3] y para una producción de 760 tdc se tiene un consumo anual de 16506492 [Nm3]. 
Tiempo de residencia y temperatura de operación del proceso de calcinación. El tiempo de residencia consiste en el periodo de tiempo que requiere la harina de crudo desde su ingreso en el cabezal de entrada del horno rotatorio hasta su paso por el cabezal de salida transformada en clinker. La temperatura de operación consiste en las temperaturas requeridas para la transformación de la harina de crudo, mismas que se distribuyen en tiempo y espacio (longitud dentro del horno). Ambas variables juegan un rol importante en la combustión de materiales que puedan ser usados como combustibles secundarios. El tiempo de residencia del fluido gaseo y del material en el proceso permitirá tener un parámetro de partida del tiempo disponible para lograr un aprovechamiento energético del AFRs.

Granulometría del material. La liberación del poder calorífico de los RS en cualquiera de los tres posibles puntos de ingreso dependerá de su granulometría.

Cantidad y calidad de RS. Se debe cuantificar la cantidad y la calidad de RS que se generan en el municipio de Sucre. El suministro de AFRs debe ser constante, garantizando la fuente energética; es necesario conocer la cantidad y los componentes de los residuos sólidos orgánicos e inorgánicos como ser: plásticos, papel, cartón, neumáticos usados, grasa animal, harina de huesos, residuos de madera, textiles.

Poder calorífico neto de los materiales. Es la cantidad de energía que puede liberar un material en la reacción química de oxidación, permitirá determinar la cantidad de combustible primario que puede ser reemplazado; valores altos involucran menor volumen requerido en la sustitución con alta reducción de los costos energéticos y de disposición de RS. Por lo general, se trabajará con un combustible heterogéneo que alcance poderes caloríficos óptimos para ser utilizados en proceso de combustión. En la Tabla 1 se presenta los poderes caloríficos individuales de diversos materiales conocidos, con datos de Karstensen (2012).

\section{Tabla 1}

Valor Calorífico Neto de AFRs

\begin{tabular}{ll}
\hline \multicolumn{1}{c}{ Combustible } & Valor Calorífico Neto [MJ/kg] \\
\hline Polietileno & 46 \\
Fuel oil ligero / Diesel (para comparación) & 42 \\
Fuelóleo pesado & 40 \\
Alquitrán & 38 \\
Grasa de animal & 37 \\
Caucho & 36 \\
Aceites usados, Residuos de refinería & 30 a 40 \\
Petcoke & 33 \\
Neumáticos Usados & 28 a 32 \\
El carbón bituminoso & 27 \\
\hline
\end{tabular}




\begin{tabular}{ll}
\hline \multicolumn{1}{c}{ Combustible } & Valor Calorífico Neto [MJ/kg] \\
\hline Polietileno & 46 \\
Fuel oil ligero / Diesel (para comparación) & 42 \\
Fuelóleo pesado & 40 \\
Alquitrán & 38 \\
Grasa de animal & 37 \\
Caucho & 36 \\
Aceites usados, Residuos de refinería & 30 a 40 \\
Petcoke & 33 \\
Neumáticos Usados & 28 a 32 \\
El carbón bituminoso & 27 \\
Líquido de sustitución Combustible & 20 a 30 \\
Plástico triturado & 18 a 22 \\
El gas de vertedero & 16 a 20 (por $\mathrm{Nm}^{3}$ ) \\
Harinas animales & 18 \\
La madera seca, la cáscara de arroz & 16 \\
Aserrín impregnado & 10 a 13 \\
Lodos de depuradora secos & 10 \\
Residuos domésticos sin clasificar & 8,5 \\
\hline
\end{tabular}

Generación de RS. Se sabe que en la gestión 2017 se generaron en promedio 160 463,82 kg/día (aprox. 160 t/d) con una cobertura de recojo de RS del $87 \%$. Se presenta la distribución por grupos generadores: domicilios $77 \%$, empresas $13 \%$, mercados $7 \%$, hospitales 3\%. La proyección para el año 2020 es de 198 t/d considerando un crecimiento poblacional de 3,7 $\%$.

Composición de los RS. Diferencia entre materia orgánica e inorgánica que permitirá determinar las cantidades posibles a usar como recurso energético, siendo de importancia para este estudio los materiales inorgánicos.

Costos de los RS. Los materiales presentes en los RS tienen un doble valor: energético y económico. Desde el punto de vista económico algunos materiales como los plásticos o papel pueden ser reciclados y ser reutilizados en industrias que requieran de esta materia prima. Los costos son los siguientes: plástico blando 2,10 BOB/Kg; plástico duro 1,20 BOB/Kg; nylon 2,10 BOB/Kg; botellas PET 1,30 BOB/Kg; aluminio $5,50 \mathrm{BOB} / \mathrm{Kg}$; y papel $1,30 \mathrm{BOB} / \mathrm{Kg}$.

RS mayores y tóxicos. Por RS se entenderá los neumáticos usados tanto de unidades pequeñas, así como de transporte pesado. En la actualidad no se está aprovechando su valor energético, pero sí se dan usos alternativos.

El tratamiento de residuos líquidos provenientes de cambio de aceite en unidades de transporte, así como aceite usado en la gastronomía tiene un tratamiento especial para su deposición en el botadero municipal de Sucre. La cantidad aproximada de aceites recolectados oscila por las $2 \mathrm{t} / \mathrm{mes}$. En los últimos años la 
Aduana Nacional ha intensificado su labor de control del contrabando registrándose confiscación de sólidos y líquidos que deben ser eliminados; el procedimiento a seguir es la destrucción y posterior deposición en los botaderos.

El análisis de las variables permitió determinar: 1) La sustitución de $20 \%$ de GN permite el co-proceso de 42,6 t/d de RS, generando un valor aproximado de energía de 20 MW para una producción de 2760 tdc; 2) La utilización de los RS generados en el municipio de Sucre se ha incrementado en los últimos años; 3) La generación de RS contiene cada vez mayores valores energéticos residuales $y$, a la vez, se complica la problemática de su administración; 4) Continuar con una eliminación de RS en botaderos municipales genera riesgos medioambientales de contaminación de tierra/venas hidrológicas por lixiviados; 5) La infraestructura existente en la planta cementera es una opción para la eliminación de los RS en el municipio de Sucre. energética en la sección de calcinación, donde se requieren altas temperaturas para transformar la materia prima en clinker, componente principal en la producción del cemento.

$\mathrm{Se}$ analiza las posibilidades técnicas $\mathrm{y}$ económicas para la implementación del coproceso de combustibles alternos en FANCESA, en busca del uso racional y optimizado de los recursos naturales y energéticos, para reducir costos y proteger el medio ambiente, específicamente mediante la reducción de la emisión de $\mathrm{CO} 2$.

\section{Análisis técnico}

El análisis se basó en el siguiente escenario de producción de clinker: un horno rotatorio, dos torres de precalentamiento DOPOL (Polysius) y SLC (FLSmidth). Capacidad de producción nominal de la línea 2000 tdc. Una distribución esquemática de la línea de producción es presentada en la Figura 1.

\section{Propuesta para el co-proceso de combustibles alternos en la cementera local}

El co-proceso en una línea de producción de cemento implica la utilización de RS como fuente

\section{Figura 1}

Flujograma horno rotatorio - torre DOPOL y SLC

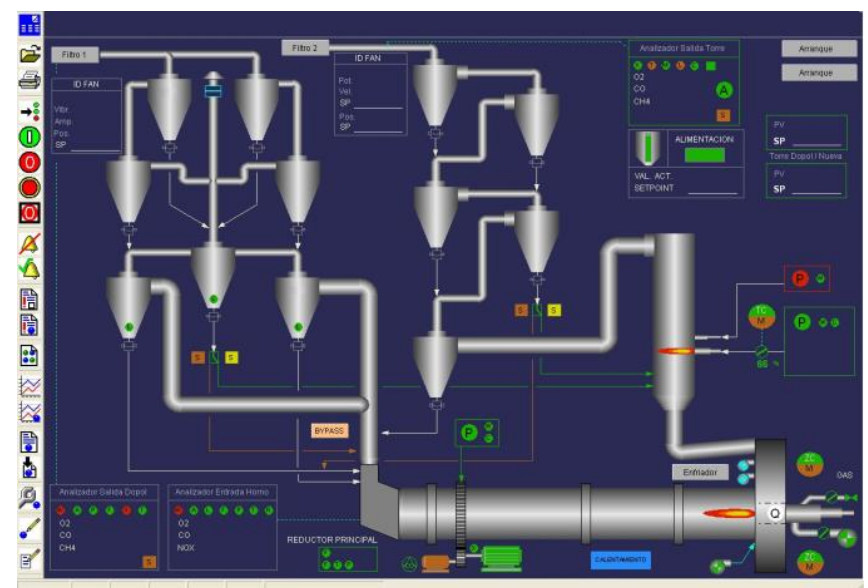


Dadas las características actuales del quemador principal, la posibilidad de uso de AFRs por este medio queda descartada. Ante ese escenario quedan dos posibles puntos de ingreso de AFRs: antes del calcinador, a través de modificaciones en el ducto terciario o en el calcinador, lo que implicaría modificaciones físicas del mismo.

\section{Pruebas de laboratorio}

La disponibilidad de equipos permitió realizar pruebas en dos niveles de temperatura: primero, llegar a temperaturas hasta los $350^{\circ} \mathrm{C}$, que son aquellas que comúnmente se alcanzan cuando se somete a un sólido a combustionar; segundo, temperaturas de operación del sistema de calcinación en las zonas más favorables, cercanas a los $900{ }^{\circ} \mathrm{C}$.

Para lograr estas temperaturas se trabajó inicialmente con un quemador Bunsen que permite someter al material a un proceso de combustión, con la característica que al material no se aplica llama directa, sino que por medio de transferencia de calor se logra que este inicie una autoignición. Sin embargo, AFRs se presentan como un compuesto heterogéneo, con componentes que no tienen el mismo punto de ignición; generando RS que requieren mayor temperatura para poder liberar su poder calorífico, lo cual no es posible con un quemador Bunsen. Esta limitante fue subsanada en un horno de inducción de laboratorio (temperatura máxima alcanzable $\left.1500{ }^{\circ} \mathrm{C}\right)$.

Con el objetivo de garantizar las calidad y estandarización del producto final, se realizaron pruebas de variación de coloración en función a la relación de AFRs y harina de crudo. Se observó variación de coloración que podría afectar en la percepción de calidad que tiene el cliente final. No se pudo generalizar los valores iniciales, relación AFRs/harina de crudo, así como resultados finales de la prueba a otro tipo de AFRs.

Al tratarse solo de pruebas iniciales con bajas cantidades de AFR no se llegó a realizar cromatografías, ni pruebas cristalográficas (equipo no disponible en cementeras nacionales) del producto final.

Preparación granulométrica de los RS. Un factor importante es lograr que los materiales a ser usados como AFR liberen todo su poder calorífico en los puntos de introducción al sistema, dejando como único residuo cenizas que no pueden ser combustionadas. Este residuo en un porcentaje será captado en filtros o saldrán con el producto final. Por lo tanto, se debe evitar RS no combustionados circulando en el sistema que puedan ocasionar obstrucciones. Esto sólo puede ser logrado si la granulometría de los AFRs está por debajo de los $30 \mathrm{~mm}$. En el presente trabajo se tuvo AFRs con dimensiones de partida de $600 \times 800 \times 900 \mathrm{~mm}, 20 \times 55 \times 85$ $\mathrm{mm}$, valores que están por encima del límite recomendado.

Para poder reducir el material dentro del rango permitido se procedió al diseño y construcción de una trituradora apta para materiales blandos. Según se observa en la Figura 2 la trituradora consta de los siguientes componentes básicos: tolva de recepción de materiales, cuchillas trituradoras, motor eléctrico de accionamiento 3 fases, tolva de recepción material triturado, ducto de salida para ingreso al sistema. 


\section{Figura 2}

Diseño de la trituradora y de las cuchillas de corte
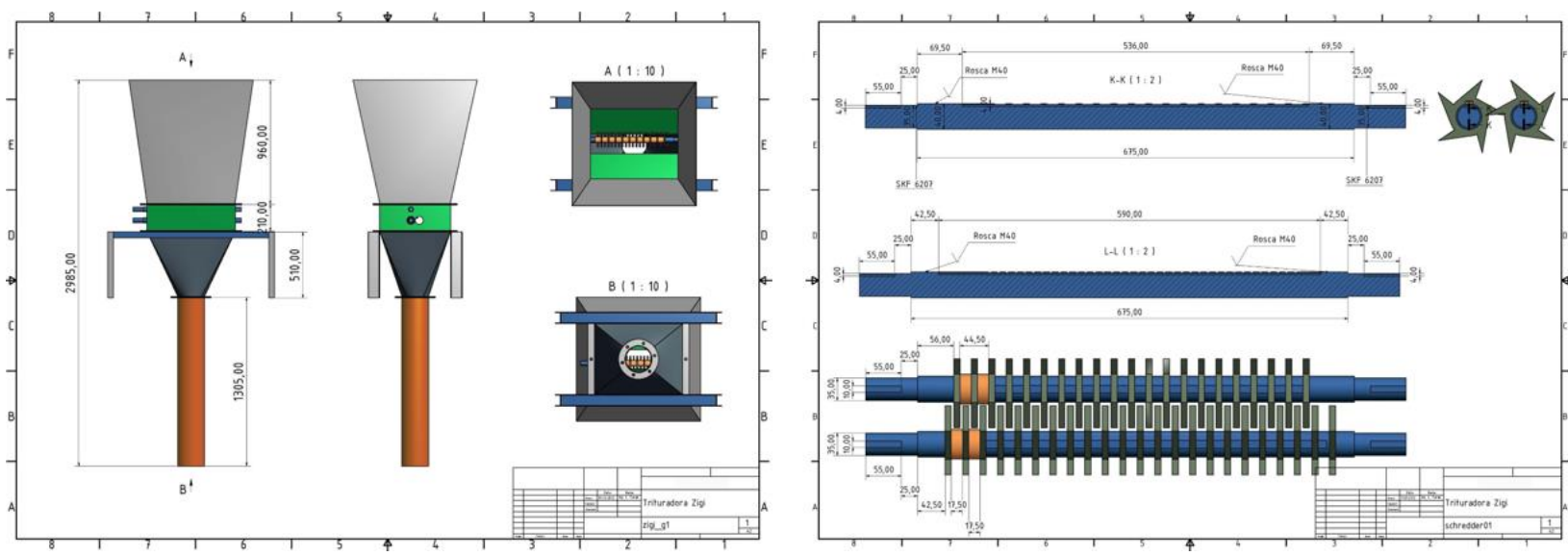

Pruebas de ingreso de RS. El sistema de calcinación, en el presente trabajo, permite el ingreso sin ninguna modificación al equipo original en sólo dos secciones: cabezal de salida del horno y ducto de aire terciario.

Primero se probó ingresando los RS por el cabezal de salida del horno, bajo las siguientes condiciones: el cabezal de salida del horno contiene el clinker; el ingreso del material permite la introducción del orden de 300 x 300 x $500 \mathrm{~mm}$, se subdividió en elementos de $300 \mathrm{x}$ $300 \times 100 \mathrm{~mm}$; las temperaturas de ingreso en esta sección llegan a un máximo de $1350{ }^{\circ} \mathrm{C}$, si bien esta temperatura permite la auto ignición del material este cae a las parrillas de enfriamiento donde la temperatura es rápidamente reducida a los $200{ }^{\circ} \mathrm{C}$ para fijar la formación de belita y evitar la formación de alita que en procesos posteriores incrementa el consumo de energía en la molienda.

Como resultado de las pruebas se tuvo dos problemas a resaltar: El producto final se ve drásticamente afectado al presentar RS; no se libera totalmente el poder calorífico, por lo tanto, el aporte energético es casi nulo.
Entonces, se probó ingresando los RS por el ducto de aire terciario, bajo las siguientes condiciones: la temperatura de circulación de los gases que provienen del cabezal de salida bordea los $900{ }^{\circ} \mathrm{C}$; estos gases calientes cruzan el calcinador donde incrementa la temperatura por la combustión del GN que se produce en el calcinador; con base en los análisis de combustión, en los cuales se determinó temperaturas de auto ignición de $350{ }^{\circ} \mathrm{C}$ y combustión completa de los residuos remanentes a temperaturas superiores a esta, el punto de ingreso por el ducto de aire terciario se ve como el punto de ingreso de AFRs más favorable; el tamaño de los AFRs no debe exceder los $30 \mathrm{~mm}$; al entrar en contacto con los gases calientes a $900{ }^{\circ} \mathrm{C}$ se procede a una auto ignición y al hacer su recorrido hacia el calcinador se tiene un tiempo de residencia de 4 - 5 segundos, suficientes como para combustionar completamente el material residual a temperaturas mayores a los $900{ }^{\circ} \mathrm{C}$; las cenizas pueden seguir dos caminos, separación del proceso por medio del Filtro By-Pass o salir con el clinker en cantidades menores. 


\section{Figura 3}

Flujo de aire terciario

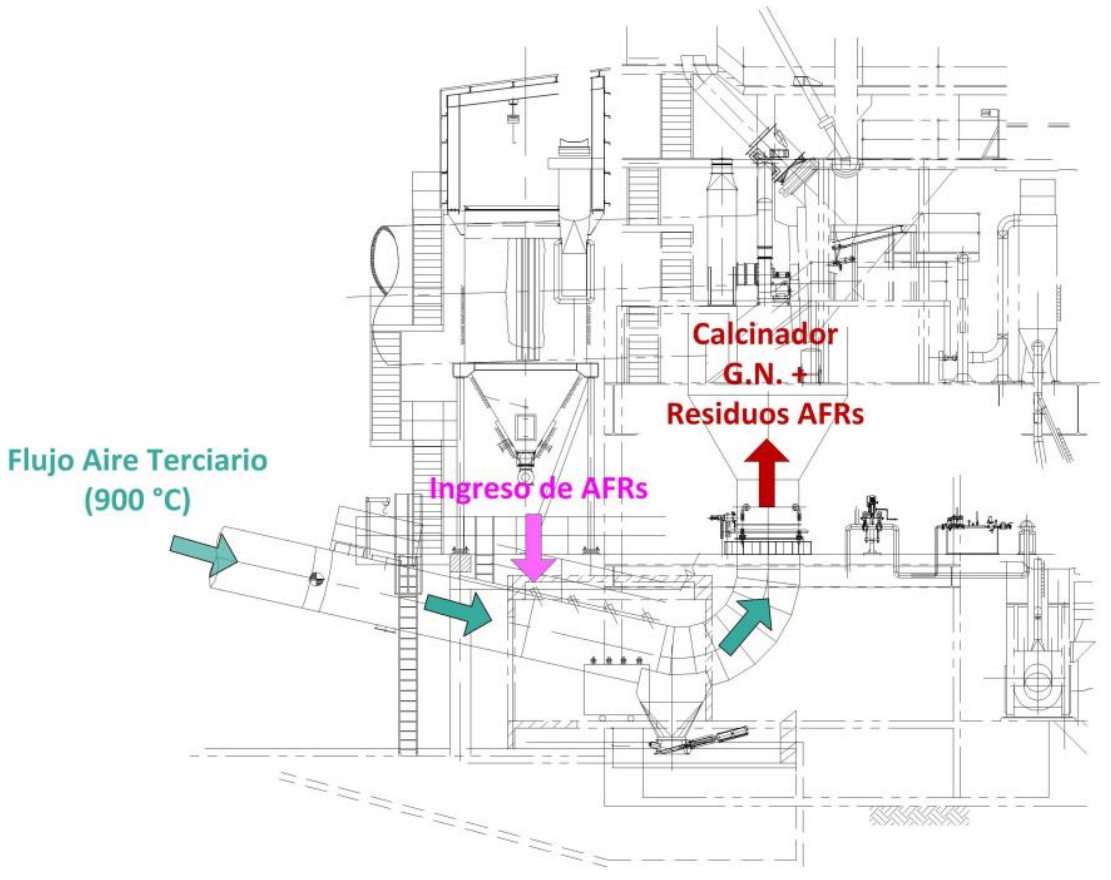

En la Figura 3 se muestra de forma esquemática el flujo de los gases calientes, así como el punto de ingreso de AFRs sin modificaciones al sistema original. Como resultado de las pruebas se consideró adecuado optar por ingresar los RS por el ducto de aire terciario.
Equipos e instalaciones requeridos. La obtención de material que pueda ser utilizado como combustible alternativo requiere un proceso previo de segregación y selección de los RS. Para este proceso se requiere una planta de selección de RS, que implicará un proceso igual al que se muestra en la Figura 4.

\section{Figura 4}

Representación gráfica de la planta de selección de RS

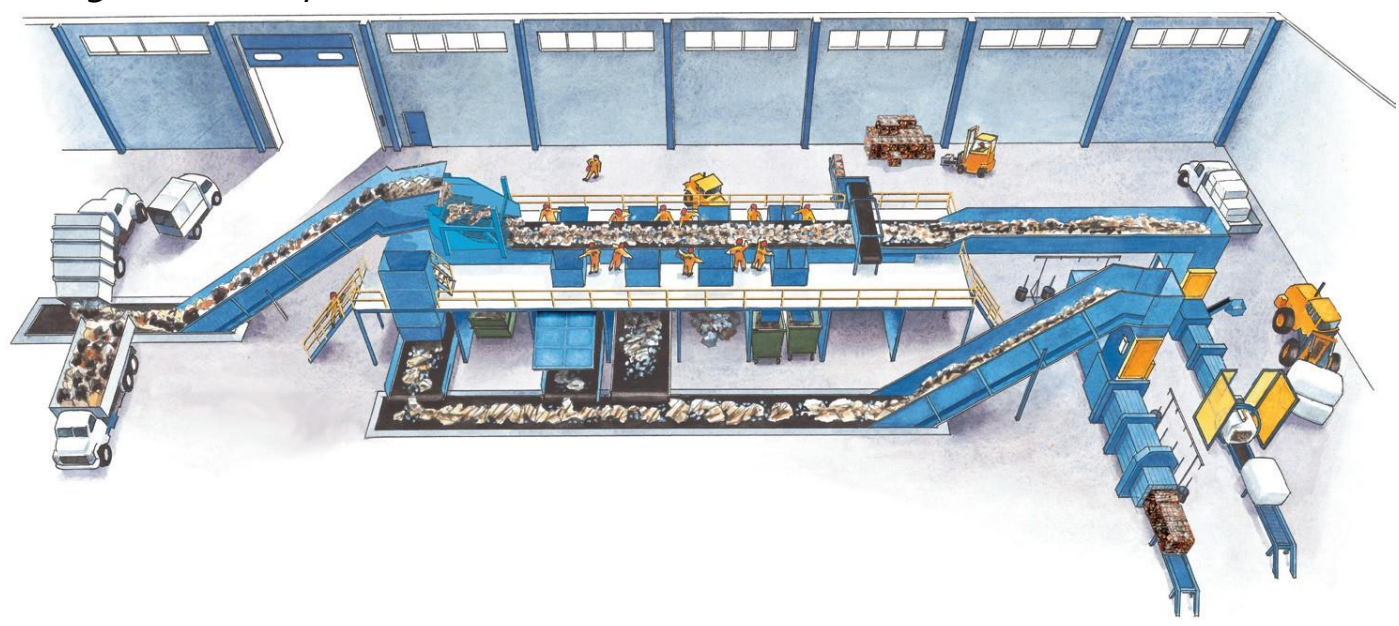


Esta nueva planta de selección y preparación de materiales deberá constar de los siguientes componentes básicos fuera y dentro de la planta: Línea de preparación de RS fuera de planta; trituradora de neumáticos usados en la planta; equipo adicional para el co-proceso en la planta.

Se estima que la inversión de la planta está entre EUR 3000000 y EUR 4500 000, según estimaciones iniciales por parte de VECOPLAN. El monto excluye instalaciones de obras civiles, así como costos de transporte e internación de la maquinaria desde Alemania hasta Sucre. La línea de separación de materiales tendrá un requerimiento estimado de 8 puestos de trabajo con una capacidad de procesamiento de $20 \mathrm{t} / \mathrm{d}$, equivalentes a aproximadamente $200 \mathrm{~kg} / \mathrm{h}$ por puesto de trabajo.

\section{Análisis Económico}

El cálculo demostrativo de los consumos y costos de GN con una sustitución de AFRs se realizó con base a los siguientes datos de partida: (a) capacidades de producción de clinker; (b) horas de trabajo 7500 h/a, equivalentes a 312 días; (c) poder calorífico de los AFRs: $18000 \mathrm{~kJ} / \mathrm{kg}$.

La producción de clinker hace referencia a las líneas de calcinación. Las 7.500 h/a contempla una parada de 45 días que normalmente es usada para efectos de mantenimiento, es decir, no existe producción de clinker en este periodo de tiempo. El poder calorífico es un promedio de una mezcla homogenizada, poderes caloríficos más altos pueden ser obtenidos en caso de usar elementos individuales (ver Tabla 1).

\section{Cálculo teórico de costos por consumo de GN}

El cálculo demostrativo de los consumos y costos de GN con una sustitución de AFRs se realizó con base en una planta de producción de $2000 \mathrm{tdc}$, con un consumo energético de $40 \%$ en el quemador principal y $60 \%$ en el quemador del calcinador. El poder calorífico es un promedio de una mezcla homogenizada, poderes caloríficos más altos pueden ser obtenidos en caso de usar elementos individuales

Cálculo de energía por hora quemador principal:

$$
2000 \frac{\mathrm{t} \cdot \mathrm{cl}}{\mathrm{dia}} \cdot 355 \frac{\mathrm{kcal}}{\mathrm{kg} \cdot \mathrm{cl}} \cdot \frac{1000}{24}=29583333 \frac{\mathrm{kcal}}{\mathrm{h}}
$$

Consumo de GN en el quemador principal:

$$
29583333 \frac{\mathrm{kcal}}{\mathrm{h}} / 9520 \frac{\mathrm{kcal}}{\mathrm{m}^{3}}=3107,49 \frac{\mathrm{m}^{3}}{\mathrm{~h}}
$$

Cálculo de energía por hora quemador calcinador:

$$
2000 \frac{\mathrm{t} \cdot \mathrm{cl}}{\mathrm{dia}} \cdot 531 \frac{\mathrm{kcal}}{\mathrm{kg} \cdot \mathrm{cl}} \cdot \frac{1000}{24}=44250000 \frac{\mathrm{kcal}}{\mathrm{h}}
$$


Consumo de GN en el calcinador considerando un $20 \%$ de sustitución de AFRs:

$$
0,2 \cdot 44250000 \frac{k c a l}{h} / 9520 \frac{k c a l}{m^{3}}=3718,49 \frac{m^{3}}{h}
$$

Gastos anuales de GN en el quemador principal:

$$
3107,49 \frac{m^{3}}{h} \cdot 0,0537 € \cdot 7500 \frac{h}{a}=1251310 €
$$

Gastos anuales de GN en el calcinador:

$$
3718,49 \frac{m^{3}}{h} \cdot 0,0537 € \cdot 7500 \frac{h}{a}=1497342 €
$$

Costos anuales de AFR substituido en el calcinador:

$$
1 \frac{€}{t} \cdot 1,7 \frac{t}{h} \cdot 7500 \frac{h}{a}=12641 €
$$

El ahorro anual estará dado por la quemador del calcinador. Bajo este escenario y diferencia de costo anual bajo una producción únicamente con GN y costo total incluida la sustitución por AFR en el sistema de calcinación resultando: Ahorro = EUR 3122 987-EUR 2761293 = EUR 361694

La producción actual de la cementera tiene un consumo energético inverso al teórico, es decir, que el $60 \%$ del GN llega a ser consumido siguiendo el procedimiento de cálculo anterior se llega a un ahorro de EUR 241811.

La segunda línea de producción con 760 tdc presenta los siguientes datos técnicos de partida: consumo energético especifico de 937 $\mathrm{kcal}=\mathrm{kg} \times \mathrm{cl}$ y consumo energético de $49 \%$ en el quemador principal y $51 \%$ en el quemador del calcinador. en el quemador principal y $40 \%$ en el

Cálculo de energía por hora quemador principal:

$$
760 \frac{\mathrm{t} \cdot \mathrm{cl}}{\mathrm{dia}} \cdot 460 \frac{\mathrm{kcal}}{\mathrm{kg} \cdot \mathrm{cl}} \cdot \frac{1000}{24}=14566667 \frac{\mathrm{kcal}}{\mathrm{h}}
$$


Consumo de GN en el quemador principal:

$$
1456667 \frac{\mathrm{kcal}}{\mathrm{h}} / 9520 \frac{\mathrm{kcal}}{\mathrm{m}^{3}}=1530,11 \frac{\mathrm{m}^{3}}{\mathrm{~h}}
$$

Cálculo de energía por hora quemador calcinador:

$$
760 \frac{t \cdot c l}{\mathrm{dia}} \cdot 477 \frac{\mathrm{kcal}}{\mathrm{kg} \cdot \mathrm{cl}} \cdot \frac{1000}{24}=15105000 \frac{\mathrm{kcal}}{\mathrm{h}}
$$

Consumo de GN en el Calcinador considerando un 20\% de sustitución de AFRs:

$$
0,2 \cdot 15105000 \frac{\mathrm{kcal}}{\mathrm{h}} / 9520 \frac{\mathrm{kcal}}{\mathrm{m}^{3}}=1269,33 \frac{\mathrm{m}^{3}}{\mathrm{~h}}
$$

Gastos anuales GN en el quemador principal:

$$
1530,11 \frac{\mathrm{m}^{3}}{h} \cdot 0,0537 € \cdot 7500 \frac{h}{a}=616138 €
$$

Gastos anuales GN en el calcinador:

$$
1269,33 \frac{m^{3}}{h} \cdot 0,0537 € \cdot 7500 \frac{h}{a}=511127 €
$$

Costos anuales de AFR substituido en el calcinador:

$$
1 \frac{€}{t} \cdot 0,7 \frac{t}{h} \cdot 7500 \frac{h}{a}=5274,00 €
$$

El ahorro anual estará dado por la diferencia de costo anual bajo una producción únicamente con GN y costo total incluida la sustitución por AFR en el sistema de calcinación resultando: Ahorro = EUR 1255 046 - EUR 1132 538,57 = EUR 122507.

El escenario actual contempla 2 líneas de producción de 2000 tdc y 760 tdc. Se tiene prevista la implementación de una nueva línea de producción, con sección de calcinación

a $18 \mathrm{~km}$ de distancia de la Planta CalOrcko. Esta nueva línea tendrá una capacidad de

$2000 \mathrm{tdc}$, con las características propias del consumo energético, es decir, $40 \%$ quemador principal y $60 \%$ quemador calcinador, siendo los resultados a obtener 
aquellos del primer análisis. Por lo tanto, el ahorro económico anual bajo el escenario actual sería de: EUR 241811 + EUR 122507 = EUR 364318.

Considerando la nueva línea de producción:

EUR 241811 + EUR 122507 + EUR 361694

$=$ EUR 726012
Cálculo de volúmenes de AFRs en el co-proceso

El volumen requerido para una sustitución inicial de $20 \%$ de GN por AFRs tiene estrecha relación con los cálculos realizados previamente.

Para una línea de producción de 2000 tdc se tiene:

$$
8850000 \frac{\mathrm{kcal}}{\mathrm{h}} /\left[5251 \frac{\mathrm{kcal}}{\mathrm{kg}} \cdot 1000\right]=1,7 \frac{\mathrm{t}}{\mathrm{h}}
$$

Por lo tanto, el volumen total de AFRs por día será del orden de:

$$
V_{A F R}=1,7 \frac{t}{h} \cdot 18 \frac{h}{d}=30 \frac{t}{d}
$$

Para una línea de producción de 760 tdc se tiene un volumen de AFRs en el calcinador de:

$$
3021,00 \frac{\mathrm{kcal}}{\mathrm{h}} /\left[4296 \frac{\mathrm{kcal}}{\mathrm{kg}} \cdot 1000\right]=0,7 \frac{\mathrm{t}}{\mathrm{h}}
$$

Por lo tanto, el volumen total de AFRs por día será del orden de:

$$
V_{A F R}=0,7 \frac{t}{h} \cdot 18 \frac{h}{d}=12,6 \frac{t}{d}
$$

En el escenario actual con una producción de 2760 tdc en el municipio de Sucre, con un $20 \%$ de sustitución inicial de GN por AFR, pueden ser co-procesados 42,6 t/d de RS. Con una nueva línea de 2000 tdc se obtendrían, teóricamente, $30 \mathrm{t}=\mathrm{d}$ adicionales de RS que pueden ser co-procesados, haciendo un total de $72,6 \mathrm{t} / \mathrm{d}$ 


\section{Costo referencial de consumo de GN}

Los costos anuales referenciales en el consumo de GN para una producción de 2760 tdc, toman como datos de partida el consumo de GN de la planta Cal Orcko (FANCESA, 2019).

\section{Cálculo del retorno de la inversión ROI}

Con una inversión inicial de EUR 4500000 en equipos requeridos para la preparación de AFRs que cumplan las condiciones para el coproceso en la industria cementera, y asumiendo EUR 5500000 necesarios para el transporte, costos de importación, maquinaria adicional (volquetas y palas e infraestructura en obras civiles); se tiene una inversión que de EUR 10000000.

Teniendo una inversión inicial de EUR 10 000000 y un ahorro anual de EUR 726012 puede ser calculado el ROI Primario:

$$
\text { ROI Primario }=\frac{10000000 €}{726012 €}=13,77 \mathrm{a}
$$

El resultado indica que la inversión se amortizará en 13 años y 9 meses. Por lo que se puede concluir que los volúmenes de RS cualitativamente como cuantitativamente permiten su uso como un combustible alternativo. Inicialmente en el ahorro de costos en la producción del cemento. Los materiales en los RS del municipio de Sucre pueden ser segregados en orgánicos e inorgánicos. La restricción de considerar a los RS como un recurso natural, involucra una segregación de materiales en papel, cartón, plásticos, neumáticos usados y metales, donde serán revalorizados para un reciclado o de acuerdo con su poder calorífico. Una vez segregados, se propone el co-proceso de material inorgánico en la planta cementera local.
Con una capacidad instalada de 2760 tdc y una proyección de 5330 tdc, en los próximos años se permitirá la eliminación por coproceso de 30 toneladas/día de RS.

El consumo volumétrico de 7,5 Millones $\mathrm{m}^{3} /$ mes de GN a un costo subvencionado de 1,70 USD/MPC, para una producción óptima de la capacidad total instalada en la cementera local, equivale a USD 450000 por mes. Un Benchmarking internacional bajo este escenario daría resultados erróneos en referencia a ahorros energéticos y desarrollo tecnológico. La industria cementera local, no ha considerado establecer nuevas estrategias empresariales de sostenibilidad, con una visión prospectiva de sustitución del GN.

El co-proceso de RS en la industria cementera local significaría un ahorro aproximado de EUR 725000 por año, a una sustitución inicial de $20 \%$ del GN, considerando el precio subvencionado del mismo con un valor energético de $20 \mathrm{MW}$. Con un precio de GN igual al precio de venta internacional fijado a 8 USD/MPC se tiene un ahorro anual de USD 3300000 .

Se calculó un ROI de 13 años y 9 meses, tiempo razonable si se toma en cuenta los beneficios sociales y la protección al medio ambiente que se obtendría. El ROI baja radicalmente su valor a menos de 4 años si se considera la eliminación de la subvención del GN.

Para garantizar el suministro constante con poderes caloríficos del orden de 22000 $\mathrm{Kcal} / \mathrm{kg}$ es necesaria una planta de selección de materiales de los RS. Para la curva de aprendizaje actual VECOPLAN calcula $20 \mathrm{t} / \mathrm{d}$ incluidas llantas usadas a ser trituradas.

La utilización de RS en la industria cementera involucra factores adicionales que deben ser considerados, como ser: desgaste de 
los ladrillos refractarios; desgaste de la virola del horno rotatorio, estabilidad óptima de producción del horno, entre otros.

Dada la cercanía del actual Botadero Municipal "Lechuguillas" a la Planta Cal-Orcko y la implementación de la nueva línea de calcinación por la Zona de la Zapatera, se deberá hacer un análisis detallado de la ubicación optima de la Planta de Manejo de RS.

\section{CONCLUSIONES}

El co-proceso permite la sustitución de hasta el $100 \%$ de combustibles fósiles no renovables. En un rango inicial entre $10 \%$ y $25 \%$, debido a la curva de aprendizaje local actual con estos combustibles; la sustitución representaría cantidades significativas en toneladas de combustibles y AFRs, permitiendo la eliminación de RS en una sinergia entre el municipio y FANCESA, que promueva el desarrollo sostenible aplicando conocimiento tecnológico; es decir, situación ganar/ganar/ganar entre la industria, la ecología y la sociedad. Esta iniciativa de un trabajo conjunto para la solución tecnológica de un problema de la sociedad puede ser replicada en otras regiones.

Este co-proceso de RS hace énfasis en el aprovechamiento energético de neumáticos usados; al respecto, los bajos volúmenes de producción iniciales pueden ayudar al proceso de curva de aprendizaje en la estabilización del horno rotatorio cuando se trabaja con este tipo de combustibles.

Para esta investigación los RS fueron considerados como fuente alternativa de energía y en ninguna instancia como material alternativo adicional para la fabricación del cemento, por lo tanto, no es necesaria una reclasificación del tipo de cemento que actualmente se produce. La implementación del co-proceso permitiría a la cementera local ser acreedora de "Bonos de Carbón" emitidos por el BID como premio a empresas que mejoren tecnológicamente la reducción de $\mathrm{CO}^{2}$. Estas están en el orden de EUR 5 a EUR 29 por tonelada de $\mathrm{CO}^{2}$. Este análisis propone un estudio intensivo de la utilización de RS en la industria cementera, específicamente: el grado de influencia en el proceso de producción de cemento, es decir, tipos y cantidades de materiales a usar, así como desgaste y fluctuaciones de producción. Asimismo, considera como base las instalaciones actuales del proceso de calcinación, es decir, la maquinaria ya instalada y que cumple con todos los requerimientos tecnológicos para el co-proceso.

Según figuras jurídicas internacionales los RS son considerados como recursos naturales y en el ámbito local se desaprovechan, siendo que estos pueden ser utilizados de una manera eficiente para la generación de energía en la industria.

Se considera que la utilización de RS que permita una autosuficiencia energética significaría un avance tecnológico en el ahorro de combustibles fósiles tradicionales y permitiría además que Sucre sea considerada una ciudad verde ecológica a niveles internacionales. Por la envergadura del presente estudio se recomienda tener a este como un punto de partida para futuros estudios en referencia a la Industria Cementera y AFRs (RS) o alternativas de generación de Energía Eléctrica en base a AFRs.

\section{REFERENCIAS}

BID. (2011). Informe Anual 2010. Banco Interamericano de Desarrollo

Braun, H. (2002). Sekundärbrennstoffeinsatz in der Zementindustrie - vom Altreifen bis 
zum Tiermehl, vol. 1. Lafarge Centre Technique Europe Centrale $\mathrm{GmbH}$

Bosold, R. D. \& Pickhardt. (2014). Zemente und ihre Herstellung. Verein Deutscher Zementwerke e.V.

CEMBUREAU. (2009). Sustainable Cement Production Co-Processing of Alternative Fuels and Raw Materials in the European Cement Industry, vol. 1

Duda, W. (1985), Internationale Verfahrenstechniken in der Zementindustrie. Bauverlag BV GmbH

EMAS. (2014). Servicio y Seguridad. Entidad municipal de aseo urbano Sucre

FANCESA. (2019). Memoria anual FANCESA 2018. Bolivia

FLSmidth. (2011). Preheater Calciner Systems. FLSmidth

FLSmidth. (2013). Pyro Processing Operations and Maintenance. FLSmidth Institute

Gante, C. H. (2007). Reduction of CO2 emissions from cement plants (Master's thesis, University of Waterloo)

Goetz-Neunhoeffer, F., Klaus, S., Neubauer, J.,
Stöber, S., Redhammer, G., Schorr, S., ... \& Beuchle, G. P. (2012). Stemmermann Reducing the $\mathrm{CO} 2$ Footprint of Inorganic

HNC. (2005). Ley de Hidrocarburos 3058. Honorable Congreso Nacional

INE, BOLIVIA. Crecimiento intercensal municipios. Instituto Nacional de Estadística, 2018

Karstensen, K. (2012). Co-processing of alternative fuels and raw materials and treatment of organic hazardous wastes in Cement kilns International experiences and best practice. SINTEF

Kropp, O. (2012). 6 KrWG - Abfallhierarchie, vol. 1. Weka Media $\mathrm{GmbH} \&$ Co. KG

Loesche.

(2009).

Loesche

Automatisierungstechnik. Loesche

Ludwig, H. (2013). Gegenwärtige und zukünftige Zemententwicklungen vor dem Hintergrund der $\mathrm{CO}_{2}$-Reduzierung. INFRAKON - Universität Weimar

Polysius. (2008). Polysius Zement Engineering made by Polysius. Polysius 


\section{Currículo de Autores}

\section{Agniezka Brissa Chavarría Hurtado}

Universidad Mayor de San Andrés, Bolivia

Licenciada en Turismo, con diplomado en Lingüística. Ha aportado desde al ámbito del turismo al Ministerio de Defensa de Bolivia. Cuenta con emprendimientos propios relacionados con turismo cultural.

\section{Luis Lemaitre Mostajo}

Banco Fassil Sociedad Anónima, Bolivia

Magister en Administración y Dirección de Empresas con diplomado en Business Intelligence. Experiencia laboral en el sector bancario, comercial y productos de consumo masivo.

Especialista en gestión herramientas de análisis de información, relevamiento y automatización de procesos a través de BPMS.

\section{Leonardo Antonio Prado Loza}

Gateway Bolivia Consultores, Bolivia

Licenciado en Turismo. Promotor e investigador turístico, especializado en sistemas de calidad turística y ecoturismo. Trabaja en ecoturismo en comunidades locales dentro de las áreas protegidas de Bolivia.

\section{Tatiana Helga Quiñones Chavarría}

Universidad Mayor de San Andrés, Bolivia

Licenciada en Turismo; con Diplomado de Educación Superior. Docente en áreas de turismo y cultura. Trabaja en turismo cultural. Actualmente cursa una licenciatura en Historia.

\section{Aleyda Rosa Reina Reyes Ortega}

Universidad San Francisco Xavier de Chuquisaca, Bolivia

Magister en sociología, con licenciatura en Trabajo Social. Docente y ponente internacional de la Universidad San Francisco Xavier de Chuquisaca. Trabajadora social, investigadora y escritora. Su accionar está orientado a la defensa de los derechos humanos y la justiciar social.. 


\section{Currículo de Autores}

\section{Luis Alberto Terán Calderón}

Universidad Andina Simón Bolívar, Bolivia

Magister en Administración de Empresas; Docente Universitario; Investigador en modelos numéricos y simulación de sistemas; Miembro del Comité Científico Técnico Departamental COVID-19 Chuquisaca. 
PORTADA 